\title{
Facilitators of and barriers to adopting a restrictive red blood cell transfusion practice: a population-based cross-sectional survey
}

\author{
Lesley J.J. Soril MSc, Tom W. Noseworthy MD MPH, Henry T. Stelfox MD PhD, \\ David A. Zygun MD MSc, Fiona M. Clement PhD
}

\section{Abstract}

Background: Despite recommendations for restrictive approaches to red blood cell transfusion in the intensive care unit (ICU), variation from best practices persists. The aim of this study was to explore potential facilitators of and barriers to practising a restrictive red blood cell transfusion strategy among intensive care physicians using the theoretical domains framework.

Methods: We conducted an online population-based cross-sectional survey of all intensive care physicians in 1 health care system (Alberta). Survey questions were based on 6 key theoretical domains of the theoretical domains framework: Knowledge, Social/professional roles and identity, Motivation and goals, Beliefs about consequences, Social influences and Beliefs about capabilities. The survey was administered between July 27 and Oct. 6, 2017. Descriptive statistics (demographic and Likert scale data) and conventional content analysis (open-ended responses) were conducted.

Results: Forty-two intensive care physicians completed the survey (estimated response rate 56\%). The respondents identified knowledge of published evidence, use of guidelines, improved outcomes, physician autonomy, and perceived culture of acceptance and collegial support as facilitators of practising a restrictive transfusion strategy. Identified barriers included potential impact on and cost to other clinical goals, conflicting practices and beliefs of physicians in other clinical specialties, deficits in medical trainees' skills and knowledge, and attitudinal barriers related to denial.

Interpretation: Using the theoretical domains framework, we identified 9 key self-reported facilitators of and barriers to intensive care physicians' transfusion behaviour. Understanding these determinants will help inform development and implementation of interventions within ICUs to encourage optimal use of red blood cell transfusion practices for nonbleeding patients whose condition is stable.

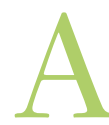

llogeneic red blood cell transfusions are common medical procedures used to treat anemia in inpatients, including those who are critically ill., ${ }^{1,2}$ Evidence-based guidelines ${ }^{3}$ and clinical recommendations from initiatives such as Choosing Wisely Canada ${ }^{4}$ recommend avoiding red blood cell transfusions for most nonhemorrhagic patients whose condition is stable and who have a hemoglobin concentration of $70 \mathrm{~g} / \mathrm{L}$ or more, as above this threshold such transfusions may be clinically inappropriate. Despite these recommendations, observational studies continue to report variation and suboptimal red blood cell transfusion practices, with pretransfusion hemoglobin concentrations of $70 \mathrm{~g} / \mathrm{L}$ or more among patients who receive transfusions worldwide. ${ }^{5-9}$ Such findings may indicate that guidelines alone are insufficient for promoting and sustaining restrictive red blood cell transfusions among physicians. However, it remains unclear why deviations from recommended best practice continue to persist and what can be done to support behaviour change.

Previous studies investigating clinical behaviour change suggest that determinants at both the individual and institu- tional levels underlie physician behaviours. ${ }^{10}$ In 2 parallel studies exploring behavioural determinants of physician transfusion practices, the theoretical domains framework was used to guide and examine qualitative findings from interviews with 28 intensive care and neonatology physicians in Canada and the United Kingdom. ${ }^{11,12}$ The theoretical domains framework is a comprehensive validated tool composed of 14 theoretically derived domains used to assess health care professionals' behaviours and inform development of techniques to prompt behaviour change. ${ }^{10,13-15}$ For local contexts seeking to promote restrictive red blood cell transfusion practices, this framework offers a reproducible means to understand facilitators and barriers, and to tailor interventional techniques. The objectives

\section{Competing interests: None declared.}

This article has been peer reviewed.

Correspondence to: Fiona Clement, fclement@ucalgary.ca

CMAJ Open 2019. DOI:10.9778/cmajo.20180209 
of this study were to explore the perceptions of red blood cell transfusion practices among intensive care physicians and to determine the key facilitators of and barriers to practising a restrictive red blood cell transfusion strategy using the theoretical domains framework.

\section{Methods}

\section{Study design and target population}

We conducted a population-based cross-sectional survey of intensive care physicians in Alberta. Eligible study participants included all physicians practising in an Alberta intensive care unit (ICU) as of July 2017. We identified potential participants through the Intensive Care Section of the Alberta Medical Association and the Alberta Health Services Critical Care Strategic Clinical Network, both of which maintain lists of intensive care physicians practising in the province.

\section{Survey development}

Two members of the research team (L.J.J.S. and F.M.C) conducted a literature review of studies exploring facilitators of and barriers to transfusion behaviour to inform questionnaire item development. Two relevant qualitative research studies were identified that used the theoretical domains framework to elucidate key factors influencing transfusion decisions among intensive care physicians across Canada ${ }^{12}$ and the UK. ${ }^{11}$ This framework synthesizes 33 theories related to behaviour and behaviour change, organized into 14 theoretical domains. ${ }^{10,13-15}$ The developers of the framework created example questions to help users assess implementation issues and health care professionals' behaviours in each domain, and a matrix of relevant behaviour change techniques. ${ }^{13,14}$ The previous qualitative studies ${ }^{11,12}$ were conducted in parallel to assess red blood cell transfusion behaviour among intensive care physicians in Canada and the UK and to compare and contrast identified determinants. Determinants relevant in both contexts were found in the following domains: Knowledge, Social/professional role and identity, Beliefs about capabilities, Beliefs about consequences, Motivation and goals, Social influences and Behavioural regulation. ${ }^{11,12}$ To leverage these extensive findings and develop an efficient and targeted survey, 3 members of the research team (L.J.J.S., T.W.N. and F.M.C.) developed initial questionnaire items to explore facilitators of and barriers to practising a restrictive red blood cell transfusion strategy within these 7 domains. Questionnaire items were also guided by interview questions posed in the previous studies ${ }^{11,12}$ and those originally developed to investigate domains of the theoretical domains framework. ${ }^{16}$ The research team reviewed all questionnaire items to ensure sufficient and nonredundant representation of items, ${ }^{17}$ and relevance to care in the ICU context. Draft items related to the Behavioural regulation domain were excluded as the information collected was found to be redundant to that obtained from the Beliefs about capabilities domain. Thus, only questions pertaining to the remaining 6 domains were developed for the final survey.
A convenience sample of 6 intensive care physicians affiliated with the critical care medicine programs of the University of Calgary and University of Alberta evaluated the survey for clinical sensibility, face validity and content validity. ${ }^{17}$ The reviewers were asked to assess the relevance and quality of each item, to identify unnecessary or ambiguous questions (including clarity, relevance to the domain, flow and wording) ${ }^{17}$ and to report the length of time required to complete the questionnaire. To assess test-retest reliability (i.e., stability), ${ }^{18}$ the reviewers were asked to complete the survey again 2 weeks later. Given that the study objective was to elucidate the facilitators of and barriers to adopting change in transfusion practice rather than to compute a score or index, the extent to which a measure is reproducible was important. ${ }^{17}$

The final survey was composed of 14 questions (presented over 7 online screens; see description of survey administration) designed to understand physician perceptions and identify self-reported behavioural determinants of practising a restrictive red blood cell transfusion strategy (i.e., restricting transfusion to patients with a hemoglobin concentration less than $70 \mathrm{~g} / \mathrm{L}$ ) (Box 1). Participants indicated their responses on a 5 -point Likert scale from 1 ("Strongly disagree") to 5 ("Strongly agree"); for the final question, the response was open-ended. We also elicited information concerning respondents' primary location of practice, number years practising in critical care, primary specialization leading to critical care and experience treating the relevant patient population. Limited demographic information was collected to decrease the likelihood of individual respondent identification.

\section{Survey administration}

Email invitations to participate in the study were sent directly from the Intensive Care Section of the Alberta Medical Association and the Critical Care Strategic Clinical Network to all eligible participants on their mailing lists. No physician contact information was shared with the research team. The invitation email included a personalized message informing participants of the purpose of the study, estimated time to complete the questionnaire, description of implied consent on survey completion, data storage and confidentiality agreement, and link to the online survey. Two reminder emails were sent within 3-week intervals after the original invitation.

The survey was administered online via SurveyMonkey (www.surveymonkey.com/) between July 27 and Oct. 6, 2017. Potential participants were informed that the survey was voluntary and was designed to understand barriers and facilitators in aggregate rather than at the individual level; therefore, the research was not intended to evaluate individual participants' knowledge or competence. ${ }^{19}$ In addition, participants were able to review and change answers (i.e., through "back" buttons) before submission. The SurveyMonkey platform tracked potential duplicate entries through IP addresses of computers used to access the survey, with no more than 1 entry permitted from a single address. However, survey responses were anonymous: IP information was not available to the research team, and no connections were made between respondent data and IP addresses. 


\section{Box 1: Final survey questions}

\section{Knowledge}

1. I am aware of the evidence in support of a restrictive red blood cell (RBC) transfusion strategy for hemodynamically stable nonbleeding adult patients in the intensive care unit (ICU).

2. The evidence base in support of a restrictive RBC transfusion strategy for hemodynamically stable nonbleeding adult patients is strong and sufficient.

3. Evidence in support of a restrictive RBC transfusion strategy guides my approach to RBC transfusions.

\section{Social/professional role and identity}

4. In general, adhering to guidelines is important to the quality of care I deliver.

5. I feel constrained by the recommendations in transfusion guidelines.

\section{Motivation and goals}

6. Adopting a restrictive $\mathrm{RBC}$ transfusion strategy can come at the cost of other goals or improving other patient outcomes (e.g., slowing time to recovery or discharge).

7. A restrictive $\mathrm{RBC}$ transfusion strategy is important to my clinical practice.

\section{Social influences}

8. I am influenced by my physician colleagues with respect to RBC transfusion practices.

9. In our ICU, my physician colleagues and I tend to practise in a similar fashion and make decisions that are consistent with the general way of agreement.

10. Patients' families have an influence on my RBC transfusion practice.

\section{Beliefs about consequences}

11. A restrictive $\mathrm{RBC}$ transfusion strategy can reduce the risk of harm (e.g., transfusion-related reactions or infections) to the patient.

12. A restrictive $\mathrm{RBC}$ transfusion strategy can reduce costs and save resources in the ICU.

\section{Beliefs about capabilities}

13. The conditions in my ICU are amenable to practising a restrictive $\mathrm{RBC}$ transfusion strategy.

14. Please elaborate on your response to question 13 :

\section{Data analysis}

We reported the total number of responses for each question and calculated percentages using the responses received as the denominator. However, only 1 question was not responded to by all participants. We analyzed the responses using descriptive statistics. For questions with Likert scale response options, the proportions of responses within each response category were depicted graphically and organized by theoretical domain of the theoretical domains framework. Openended response data were analyzed by 2 researchers (L.J.J.S. and F.M.C.) using conventional qualitative content analysis, ${ }^{20}$ a method typically used to describe a phenomenon when existing evidence is limited..$^{20} \mathrm{We}$ took an inductive approach to develop an initial list of emergent themes for why respondents perceived their ICU contexts to be (or not be) amenable to practising a restrictive transfusion strategy. The most prominent or key themes and interpretation of the data were then determined through discussion and consensus among all members of the team.

\section{Ethics approval}

Research ethics approval was obtained from the Conjoint Health Research Ethics Board of the University of Calgary.

\section{Results}

Of the estimated 75 intensive care physicians practising in the province, 42 completed the survey (response rate 56\%). The majority of respondents identified a large urban hospital as their primary location of practice (21/39 [54\%]), had over 15 years of experience practising in critical care (23/41 [56\%]) and reported medicine as the clinical specialty that led them to critical care medicine (24/40 [60\%]) (Table 1). Most strongly agreed $(37 / 42[88 \%])$ or agreed $(4 / 42[10 \%])$ that they commonly encounter patients with borderline hemoglobin concentrations (70-90 g/L), for whom a restrictive transfusion strategy could apply; 1 respondent (2\%) neither agreed nor disagreed.

\section{Determinants of practising a restrictive red blood cell transfusion strategy}

The responses on the Likert scale survey questions evaluating potential facilitators of and barriers to practice change, grouped by theoretical domain, are provided in Figure 1.

\section{Identified facilitators}

Respondents identified 2 major facilitators: knowledge of the evidence in support of restrictive red blood cell transfusions and use of transfusion guidelines. Almost all respondents

Table 1: Characteristics of survey respondents

\begin{tabular}{|lc|}
\hline Characteristic & $\begin{array}{c}\text { No. }(\%) \text { of } \\
\text { respondents } \\
n=42\end{array}$ \\
\hline Primary location of practice $(n=39)$ & $18(46)$ \\
\hline Teaching hospital & $21(54)$ \\
\hline$\quad$ Large urban hospital & \\
\hline $\begin{array}{l}\text { No. of years practising in critical care } \\
(n=41)\end{array}$ & $2(5)$ \\
\hline$<5$ & $7(17)$ \\
\hline $5-9$ & $9(22)$ \\
\hline $10-14$ & $23(56)$ \\
\hline$\geq 15$ & \\
\hline $\begin{array}{l}\text { Clinical specialty leading to critical care } \\
(n=40)\end{array}$ & $24(60)$ \\
\hline Medicine & $6(15)$ \\
\hline Surgery & $5(12)$ \\
\hline Anesthesia & $5(12)$ \\
\hline Other & \\
\hline
\end{tabular}



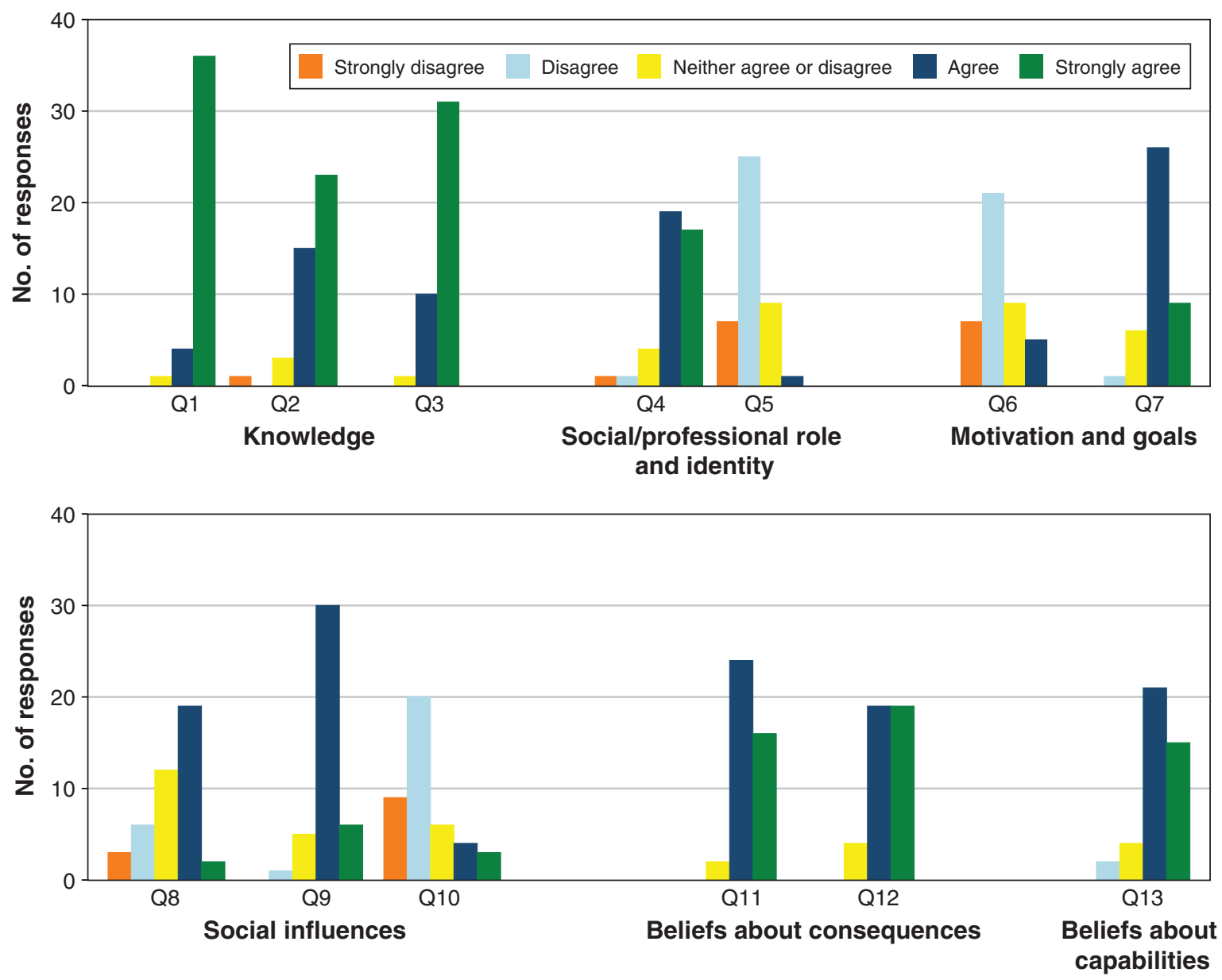

Figure 1: Summary of Likert scale survey responses by theoretical domain. Note: $Q=$ question.

reported being aware of the evidence (36/41 [88\%] strongly agreed, 4/41 [10\%] agreed), and over 90\% (23/42 [55\%]) reported that it was strong and sufficient (Knowledge domain; questions 1 and 2). Most respondents (31/42 [74\%] strongly agreed, 10/42 [24\%] agreed) reported using the evidence to guide their practice (Knowledge domain; question 3). Moreover, 36/42 respondents (86\%) felt that guidelines are important to the quality of care, and 32/42 (76\%) did not feel constrained by transfusion guidelines (25 [60\%] strongly disagreed, 7 [17\%] disagreed) (Social/professional role and identity domain). Perceived improvements in both patient and health care system outcomes (Beliefs about consequences domain) were also identified as key facilitators: most respondents agreed that a restrictive red blood cell transfusion strategy could reduce the risk of patient harm (16/42 [38\%] strongly agreed, $24 / 42$ [57\%] agreed), reduce costs and save resources in the ICU (19/42 [45\%] strongly agreed, 19/42 [45\%] agreed).

Most respondents (15/42 [36\%] strongly agreed, 21/42 [50\%]) agreed) reported that their ICUs were amenable to practising a restrictive red blood cell transfusion strategy
(Beliefs about capabilities domain). In the open-ended elaboration on these responses, respondents identified 2 facilitators centred on the dynamics between health care providers within the ICU: physician autonomy in clinical decision-making (Beliefs about capabilities domain), and a culture of acceptance (i.e., of restrictive transfusions) and collegial support (Environmental context and resources domain) (Table 2). Other facilitators described by respondents included implementation of strategies and approaches to encourage uptake of a restrictive transfusion strategy (Behavioural regulation domain), including the use of transfusion practice guidelines (Social/professional role and identity domain), and a multidisciplinary approach to health care provider education (Knowledge domain).

\section{Identified barriers}

Respondents identified the potential impacts on other areas of practice or goals as barriers to practising a restrictive transfusion strategy. Five respondents (12\%) agreed that adopting a restrictive red blood cell transfusion strategy could come at the cost of other clinical and/or patient goals (e.g., slowing 
Table 2: Thematic analysis of respondents' open-ended responses elaborating on amenability of intensive care unit to practising a restrictive transfusion strategy

\begin{tabular}{|c|c|c|}
\hline Theme & $\begin{array}{l}\text { Relevant theoretical domains } \\
\text { framework domain }\end{array}$ & Illustrative quote \\
\hline \multicolumn{3}{|l|}{ Facilitators } \\
\hline Physician autonomy & Beliefs about capabilities & "There is relative freedom in decision-making." [R5] \\
\hline $\begin{array}{l}\text { Culture of acceptance and } \\
\text { collegial support }\end{array}$ & $\begin{array}{l}\text { Environmental context and } \\
\text { resources }\end{array}$ & $\begin{array}{l}\text { "The [physician] group has pretty consistent practices, so if [an] } \\
\text { approach is adopted, it tends to be followed." [R40] } \\
\text { "Culture that accepts restrictive transfusion strategy." [R15] }\end{array}$ \\
\hline $\begin{array}{l}\text { Multidisciplinary approach to } \\
\text { education }\end{array}$ & Knowledge; behavioural regulation & $\begin{array}{l}\text { "Multidisciplinary education surrounding guidelines for transfusion } \\
\text { have diminished requests for transfusion when not indicated." [R6] }\end{array}$ \\
\hline Use of clinical guidelines & $\begin{array}{l}\text { Social/professional role and } \\
\text { identity; behavioural regulation }\end{array}$ & $\begin{array}{l}\text { "We have a local guideline supporting restrictive red blood cell } \\
\text { transfusion practices." [R26] }\end{array}$ \\
\hline \multicolumn{3}{|l|}{ Barriers } \\
\hline $\begin{array}{l}\text { Conflict between clinical } \\
\text { specialties }\end{array}$ & Social influences & $\begin{array}{l}\text { "No significant barriers among intensivists, but consulting services } \\
\text { at times at odds." [R29] }\end{array}$ \\
\hline $\begin{array}{l}\text { Knowledge and skills of } \\
\text { medical trainees }\end{array}$ & Knowledge; skills & $\begin{array}{l}\text { "Our [intensive care unit] is staffed by residents at night, and they } \\
\text { don't always adhere to restrictive transfusion strategies." [R12] }\end{array}$ \\
\hline Perceived nonissue & Optimism & $\begin{array}{l}\text { "This [is] really NOT a problem, so why bother expanding on an } \\
\text { answer to a question that did not need to be asked?" [R11] }\end{array}$ \\
\hline
\end{tabular}

time to recovery or discharge from the ICU), (Motivation and goals), whereas 9 respondents (21\%) neither agreed nor disagreed with this statement.

Six $(14 \%)$ of the 42 respondents did not perceive that or were indifferent to whether their ICU was amenable to practising a restrictive transfusion strategy. Analysis of open-ended responses elaborating on these responses identified 3 additional barriers (Table 2). Although half of the respondents reported that they were not influenced by or were indifferent to their ICU physician colleagues with respect to red blood cell transfusion (Social influences domain), perceived conflicts between ICU health care providers and other clinical specialties was seen as a barrier to practising a restrictive strategy. Another barrier related to the nascent skills and knowledge of medical trainees and how this may hinder their ability to consistently adhere to a restrictive transfusion practice. A final attitudinal barrier that emerged was the perception that variability in red blood cell transfusion practices did not occur (Optimism domain).

\section{Interpretation}

We report on how intensive care physicians in Alberta perceive current red blood cell transfusion practices in the ICU and factors that influence restrictive red blood cell transfusion practices for nonbleeding adult patients whose condition is stable. Key facilitators identified included respondents' perceived knowledge of the evidence in support of restrictive red blood cell transfusions, reported use of transfusion guidelines, potential improvements in patient and system outcomes, physician autonomy, and a perceived culture of acceptance and collegial support in the ICU. Barriers included the potential impact on and cost to other clinical goals, conflicting practices and beliefs of physicians in other clinical specialties, deficiencies in residents' skills and knowledge, and the perception that variation in red blood cell transfusion practices (e.g., nonadherence to guidelines) does not exist. Our findings are in line with the facilitators and barriers previously identified in the literature. ${ }^{11,12}$ For instance, one barrier for our respondents concerned conflicts with physicians in medical specialties outside of the ICU (Social influences domain). Given the interdisciplinary care provided to critically ill patients, ${ }^{21}$ differences in adopted transfusion best practices across specialties may impede implementation of ICU recommendations and/or guidelines.

Open-ended responses also identified facilitators and barriers in additional theoretical domains. One barrier was associated with the Skills domain and related to the abilities of medical trainees. This echoes findings from a previous survey study that showed insufficient medical resident knowledge of and training in transfusion medicine. ${ }^{22}$ However, such observations may also result from variability in the transfusion standards disseminated to trainees by physician educators in different medical specialties. Another barrier we identified related to the Optimism domain: 1 respondent did not perceive variability in red blood cell transfusion practices in the ICU and felt that exploration into this area was unwarranted. This finding may represent an important attitudinal barrier, particularly if expressed by physicians in a leadership role.

\section{Limitations}

Our respondents consisted of a small sample of intensive care physicians from 1 Canadian province. Although the observed nonresponse rate, $44 \%$, is considered reasonable for electronic surveys, ${ }^{17}$ it may indicate nonresponse bias. ${ }^{23}$ Our results may also have been affected by selection bias through undercoverage or voluntary response bias, as we were unable to determine the true number of eligible intensive care physicians in 
Alberta. ${ }^{24,25}$ To build off previous research, ${ }^{11,12}$ we designed our survey to explore potential facilitators and barriers in 6 of the 14 domains in the theoretical domains framework (Knowledge, Social/professional roles and identity, Motivation and goals, Beliefs about consequences, Social influences and Beliefs about capabilities). Although these 6 domains were among the most relevant to red blood cell transfusion behaviours of Canadian and UK intensive care physicians, ${ }^{11,12}$ this choice prevented us from extensively exploring determinants in the other 8 theoretical domains framework domains. We examined the stability and face and content validity of the survey through pilot testing; however, evaluation of other psychometric properties (e.g., internal consistency) was not completed. This may have affected the reliability and validity of the present findings, although the extent of such impact is unclear. Our findings may be subject to duplicate survey entries. The SurveyMonkey platform tracked unique respondents through computer IP addresses, and duplicate entries may have resulted if respondents used different computers; however, the likelihood of this is low. All of our respondents practised primarily in teaching hospitals or hospitals in large urban settings, where most ICUs in Alberta are located. Thus, the generalizability of our findings to intensive care physicians in other geographic settings is unknown. Last, the crosssectional nature of the study permitted us to understand physician perceptions at only 1 point in time; physician perceptions may evolve over time.

\section{Conclusion}

We identified several potential facilitators of and barriers to adopting a restrictive red blood cell transfusion practice among intensive care physicians in Alberta. This information can be used to develop targeted behaviour change techniques to optimize red blood cell transfusion practices in the ICU. This theory-based, methodological approach for soliciting behavioural determinants to transfusion behaviours may also apply in other clinical contexts.

\section{References}

1. Carson JL, Noveck H, Berlin JA, et al. Mortality and morbidity in patients with very low postoperative $\mathrm{Hb}$ levels who decline blood transfusion. Transfusion 2002;42:812-8.

2. Weiskopf RB, Beliaev AM, Shander A, et al. Addressing the unmet need of lifethreatening anemia with hemoglobin-based oxygen carriers. Transfusion 2017; 57:207-14.

3. Carson JL, Guyatt G, Heddle NM, et al. Clinical practice guidelines from the AABB: red blood cell transfusion thresholds and storage. $7 A M A$ 2016;316: 2025-35.

4. Canadian Critical Care Society; Canadian Association of Critical Care Nurses; Canadian Society of Respiratory Therapist. Five things clinicians and patients should question. Available: https://choosingwiselycanada.org/wp-content/ uploads/2017/04/Critical-care.pdf (accessed 2019 Feb. 25).

5. Sadeghi A, Belali S, Ali Asgari A, et al. Inappropriate packed RBC transfusion in a tertiary care center. Arch Iran Med 2017;20:83-5.

6. Corwin HL, Gettinger A, Pearl RG, et al. The CRIT Study: anemia and blood transfusion in the critically ill — current clinical practice in the United States. Crit Care Med 2004;32:39-52.
7. Chohan SS, McArdle F, McClelland DB, et al. Red cell transfusion practice following the Transfusion Requirements in Critical Care (TRICC) study: prospective observational cohort study in a large UK intensive care unit. Vox Sang 2003;84:211-8

8. Netzer G, Liu X, Harris AD, et al. Transfusion practice in the intensive care unit: a 10-year analysis. Transfusion 2010;50:2125-34.

9. Surial B, Burkhart A, Terliesner N, et al. Adherence to transfusion guidelines: Are we prepared for the Smarter Medicine or Choosing Wisely initiative? Swiss Med Wkly 2015;145:w14084.

10. Cane J, O'Connor D, Michie S. Validation of the theoretical domains framework for use in behaviour change and implementation research. Implement Sci 2012;7:37.

11. Francis JJ, Stockton C, Eccles MP, et al. Evidence-based selection of theories for designing behaviour change interventions: using methods based on theoretical construct domains to understand clinicians' blood transfusion behaviour. Br 7 Health Psychol 2009;14:625-46.

12. Islam R, Tinmouth AT, Francis JJ, et al. A cross-country comparison of intensive care physicians' beliefs about their transfusion behaviour: a qualitative study using the theoretical domains framework. Implement Sci 2012;7:93.

13. Michie S, Johnston M, Abraham C, et al.; "Psychological Theory" Group. Making psychological theory useful for implementing evidence based practice: a consensus approach. Qual Saf Health Care 2005;14:26-33.

14. Michie S, Johnston M, Francis J, et al. From theory to intervention: mapping theoretically derived behavioural determinants to behaviour change techniques. Appl Psychol 2008;57:660-80.

15. Atkins L, Francis J, Islam R, et al. A guide to using the theoretical domains framework of behaviour change to investigate implementation problems. Implement Sci 2017;12:77.

16. Michie S, Johnston M, Abraham C, et al.; "Psychological Theory" Group. Making psychological theory useful for implementing evidence based practice: a consensus approach. Qual Saf Health Care 2005;14:26-33.

17. Burns KE, Duffett M, Kho ME, et al.; ACCADEMY Group. A guide for the design and conduct of self-administered surveys of clinicians. CMA7 2008;179: $245-52$

18. Souza AC, Alexandre NMC, Guirardello EB. Psychometric properties in instruments evaluation of reliability and validity. Epidemiol Serv Saude 2017;26: 649-59.

19. Veloski J, Tai S, Evans AS, et al. Clinical vignette-based surveys: a tool for assessing physician practice variation. Am J Med Qual 2005;20:151-7.

20. Hsieh HF, Shannon SE. Three approaches to qualitative content analysis. Qual Health Res 2005;15:1277-88.

21. James FR, Power N, Laha S. Decision-making in intensive care medicine - a review. Z Intensive Care Soc 2018;19:247-58.

22. Kasraian L, Tavassoli A. A survey of resident physicians' knowledge concerning transfusion medicine in Shiraz, Iran. Asian $\mathcal{F}$ Transfus Sci 2014;8:118-20.

23. Johnson TP, Wislar JS. Response rates and nonresponse errors in surveys. 7AMA 2012;307:1805-6.

24. Leece P, Bhandari $M$, Sprague S, et al. Internet versus mailed questionnaires: a randomized comparison (2). 7 Med Internet Res 2004;6:e30.

25. Passmore C, Dobbie AE, Parchman M, et al. Guidelines for constructing a survey. Fam Med 2002;34:281-6.

Affiliations: Departments of Community Health Sciences (Soril, Noseworthy, Stelfox, Clement) and Critical Care Medicine (Stelfox), Cumming School of Medicine, and O'Brien Institute for Public Health (Soril, Noseworthy, Stelfox, Clement), University of Calgary, Calgary, Alta.; Department of Critical Care Medicine (Zygun), Alberta Health Services; Faculty of Medicine and Dentistry (Zygun), University of Alberta, Edmonton, Alta.

Contributors: Lesley Soril analyzed the data. Lesley Soril and Fiona Clement drafted the manuscript. All of the authors contributed to the study conception and design, and data interpretation, critically revised the manuscript for important intellectual content, approved the version to be published and agreed to be accountable for all aspects of the work.

Funding: Lesley Soril is supported by an Alberta Innovates - Health Solutions Graduate Studentship Award.

Supplemental information: For reviewer comments and the original submission of this manuscript, please see www.cmajopen.ca/content $/ 7 / 2 /$ E252/suppl/DC1. 